\title{
MicroRNA-200c affects bladder cancer angiogenesis by regulating the Akt2/mTOR/HIF-1 axis
}

\author{
Shui-Qing Wu, Hai-Qing He, Ye Kang, Ran Xu, Lei Zhang, Xiao-Kun Zhao, Xuan Zhu \\ Department of Urology, The Second Xiangya Hospital of Central South University, Changsha 410011, China \\ Contributions: (I) Conception and design: SQ Wu, X Zhu; (II) Administrative support: X Zhu, L Zhang, XK Zhao; (III) Provision of study materials \\ or patients: SQ Wu, HQ He, Y Kang; (IV) Collection and assembly of data: HQ He, Y Kang; (V) Data analysis and interpretation: L Zhang, XK \\ Zhao; (VI) Manuscript writing: All authors; (VII) Final approval of manuscript: All authors. \\ Correspondence to: Dr. Xuan Zhu. Department of Urology, The Second Xiangya Hospital of Central South University, No. 139, Middle Renmin Road, \\ Changsha 410011, China. Email: zhuxuan@csu.edu.cn.
}

\begin{abstract}
Background: Bladder cancer is one of the most frequent urologic tumours in the world. MicroRNA200c (miR-200c) has been considered a regulator of tumour angiogenesis. Akt2/mTOR was considered a regulator of vascular endothelial growth factor (VEGF) and hypoxia-inducible factor $1 \alpha(\mathrm{HIF}-1 \alpha)$. However, the mechanism by which miR-200c regulates bladder cancer angiogenesis remains unknown.

Methods: Western blotting and qRT-PCR were used to detect the expression of protein and mRNA, respectively. Cell proliferation, migration and invasion were detected using MTT, wound-healing and transwell assays, respectively. A dual luciferase reporter assay was used to identify the binding site between miR-200c and Akt2. A tube formation assay was also applied to detect the angiogenesis ability.

Results: Significantly higher expression levels of HIF-1 $\alpha$ and VEGF and lower levels of miR-200c were observed in three types of bladder cancer cell lines. Transfection with the miR-200c mimic markedly inhibited cell viability, angiogenesis, and the expression of VEGF and HIF-1 $\alpha$. Overexpression of miR-200c remarkably suppressed the expression of Akt2, and the binding site between them was identified. Knockdown of Akt2 remarkably decreased the expression of VEGF and HIF-1 $\alpha$ by regulating mTOR. miR-200c influenced the expression of VEGF and HIF-1 $\alpha$ through the Akt2/mTOR signalling pathway and further regulated angiogenesis in bladder cancer cells.

Conclusions: We proved that miR-200c could suppress HIF-1 $\alpha$ /VEGF expression in bladder cancer cells and inhibit angiogenesis, and these regulations were achieved by targeting Akt $2 / \mathrm{mTOR}$. This study may provide new insight into the prevention and treatment of bladder cancer.
\end{abstract}

Keywords: MicroRNA-200c; bladder cancer; angiogenesis; Akt2/mTOR/HIF-1 $\alpha$

Submitted Mar 06, 2019. Accepted for publication Oct 10, 2019.

doi: $10.21037 /$ tcr.2019.10.23

View this article at: http://dx.doi.org/10.21037/tcr.2019.10.23

\section{Introduction}

Bladder cancer is one of the most common malignant tumours in the urinary system and has a complex pathogenesis (1). At present, the clinical incidence of bladder cancer is increasing every year (2). Bladder cancer, like other cancers, is associated with a substantial amount of angiogenesis during the growth of tumours (3). Clinical studies have shown that inhibiting angiogenesis can effectively inhibit the growth of tumours (4).
MicroRNAs (miRNAs) are small non-coding RNAs that mediate related target genes by binding to the $3^{\prime}$ untranslated regions of the protein-coding transcripts (5). It was reported that they play an important role in tumourigenesis, progression and migration of tumours $(6,7)$. MicroRNA200c (miR-200c) has been considered a regulator during the progression of endometrial (8) and pancreatic cancer (9). Meanwhile, miR-200c could regulate the EMT process and further suppress the invasion and proliferation of bladder 
cancer cells (10). We previously found that microRNA200c (miR-200c) is differentially expressed in bladder cancer. Meanwhile, it is associated with the deterioration of bladder cancer $(5,11)$ and is also involved in angiogenesis $(9)$. However, the mechanism of its involvement in the angiogenesis of bladder cancer is unclear.

Akt, a serine/threonine kinase, plays a key role in tumourigenesis, and Akt2, a pro-survival protein, is a member of the Akt family $(12,13)$. According to database queries and literature reports, Akt2 is viewed as a direct target of miR-200c $(11,14)$. Akt $2 / \mathrm{mTOR}$ has been considered an important pathway influencing the angiogenesis progression of cancers $(15,16)$. Meanwhile, Akt2/mTOR can regulate the level of vascular endothelial growth factor (VEGF) (17), and miR-200c may regulate the angiogenesis of bladder cancer by targeting Akt2. It was demonstrated that the hypoxia-inducible factor $1 \alpha(\mathrm{HIF}-1 \alpha) / \mathrm{VEGF}$ signalling pathway plays a key role in tumour angiogenesis $(18,19)$, and Akt/mTOR could influence the expression of HIF-1 $\alpha(20,21)$. However, the modulation of $\mathrm{Akt} / \mathrm{mTOR}$ in bladder cancer has not been reported.

In this study, we found significantly lower expression of miR-200c in bladder cancer cells compared with control cells. The upregulation of miR-200c could inhibit Akt2 and influence the $\mathrm{Akt} 2 / \mathrm{mTOR}$ pathway, resulting in the suppression of the HIF- $1 \alpha / \mathrm{VEGF}$ axis. Therefore, miR200c may inhibit the proliferation of bladder cancer cells and angiogenesis through the method described above, and the present study may provide novel insight for the prevention and treatment of bladder cancer.

\section{Methods}

\section{Cell culture}

In this study, the SV-HUC-1 (human bladder epithelial cells), 5637 (human bladder cancer cells), T24 (human bladder cancer cells), and MB49 (mouse bladder cancer cells) cell lines were obtained from American Type Culture Collection (Rockville, MA, USA). The cells were cultured normally in DMEM (Gibco, USA) with 10\% FBS (Gibco, USA) at $37{ }^{\circ} \mathrm{C}$ in a humidified atmosphere containing $5 \%$ $\mathrm{CO}_{2}$. The reagents Rapamycin (activator of $\mathrm{mTOR}$ ) and MHY1485 (inhibitor of mTOR) used to treat cells were purchased from Sigma (St. Louis, USA)

\section{Cell transfection}

The miR-200c mimic, miR-200c inhibitor, pcDNA-Akt2, and sh-Akt 2 were designed and synthesized by GenePharma Co., Ltd (Shanghai, China). Cells were seeded on 60-mm dishes and cultured for $24 \mathrm{~h}$. After $70 \%$ cell confluence was achieved, cell transfection and co-transfection were applied using Lipofectamine 2000 (Invitrogen). Lipofectamine 2000 suspension was prepared with $5 \mu \mathrm{L}$ of Lipofectamine 2000 and $250 \mu \mathrm{L}$ of Opti-MEM. After incubation with Lipofectamine 2000 suspension for $6 \mathrm{~h}$ at $37^{\circ} \mathrm{C}$ in a humidified atmosphere containing $5 \% \mathrm{CO}_{2}$, the transfection efficiency was measured, and the medium was replaced. Cells were collected after $48 \mathrm{~h}$ of incubation at $37^{\circ} \mathrm{C}$ and $5 \% \mathrm{CO}_{2}$.

\section{$m R N A$ extraction and quantitative real-time $P C R$ (qRT-PCR)}

First, RNA was extracted using TRIzol reagent (Invitrogen Life Technologies, USA). A total of $500 \mathrm{ng}$ of RNA was reverse-transcribed into cDNA using the Primer Script RT reagent kit (Takara Bio, China). Real-time PCR was performed using the SYBR Premix Ex TaqTM II kit (Takara Bio, China). Amplification conditions were conducted as follows: $95{ }^{\circ} \mathrm{C}$ for $10 \mathrm{~min}$, followed by 45 cycles consisting of $95^{\circ} \mathrm{C}$ for $15 \mathrm{~s}, 60{ }^{\circ} \mathrm{C}$ for $30 \mathrm{~s}$ and $68{ }^{\circ} \mathrm{C}$ for $60 \mathrm{~s}$. The relative mRNA level was analysed by the $2^{-\Delta \Delta \mathrm{Ct}}$ method. The primers used for miR-200c, HIF-1 $\alpha$, VEGF, and Akt2 were as follows: mus-miR-200c-forward 5'-GCCCGCTAATACTGCCGGGTAAT-3', reverse 5'-GTGCAGGGTCCGAGGT-3'; mus-HIF-1 $\alpha$-forward 5'-GTCCCAGCTACGAAGTTACAGC-3', reverse 5'-CAGTGCAGGATACACAAGGTTT-3'; mus-VEGFforward 5'-GCACCCACGACAGAAGGA-3', reverse 5'-GCTTCGCTGGTAGACATCCAT-3'; hsa-miR-200cforward 5'-TAATACTGCCGGGTAATGATGGA-3', reverse 5'-TGGTGTCGTGGAGTCG-3'; hsa-HIF-1 $\alpha$ forward 5'-TCAAAGTCGGACAGCCTCA-3', reverse 5'-CCCTGCAGTAGGTTTCTGCT-3'; hsa-VEGFforward 5'-GAAGTGGTGAAGTTCATGGATGTC-3', reverse 5'-CGATCGTTCTGTATCAGTCTTTCC-3'; Akt2-forward 5'-GAGGTCATGGAGCACAGGTT-3', reverse 5'-CTGGTCCAGCTCCAGTAAGC'; musU6-forward: 5'-CTCGCTTCGGCAGCACA-3', reverse: 5'-AACGCTTCACGAATTTGCGT-3'; hsa-Uforward: 5'-CTTCGGCAGCACATATAC-3', reverse: 5'-GAACGCTTCACGAATTTGC-3'; mus- $\beta$-actinforward: 5'-GGCTGTATTCCCCTCCATC-3', reverse: 5'-ATGCCATGTTCAATGGGGTA-3'; hsa- $\beta$-actinforward: 5'-TGGCACCCAGCACAATGAAGA-3', reverse: 5'-GAAGCATTTGCGGTGGACGAT-3'. Every sample 
was replicated 3 times with no RT and no template controls. Data were analysed by comparing cycle threshold values. The relative expression of target genes was calculated using the $2^{-\Delta \Delta \mathrm{Ct}}$ method. $\Delta \Delta \mathrm{Ct}=\Delta \mathrm{Ct}_{\text {experiment }}-\Delta \mathrm{Ct}_{\text {control }}, \Delta \mathrm{Ct}=\mathrm{Ct}_{\text {target }}$ gene $-\mathrm{Ct}_{\text {control gene }}$. The fold change between the experimental group and the control group $=2^{-\Delta \Delta \mathrm{Ct}}$.

\section{Western blot analysis}

Primary antibodies used in this study, including HIF-1 $\alpha$, VEGF, Akt2, mTOR, and p-mTOR, were obtained from Abcam (Hong Kong, China). Cells were collected and lysed in RIPA buffer (Sigma-Aldrich, USA). Protein concentrations were measured with the BCA protein assay kit (Thermo Fisher Scientific, USA). Equal amounts of protein were loaded on an SDS-PAGE gel, and then the proteins were transferred to a PVDF membrane (Millipore, Billerica, MA, USA). Skimmed milk (5\%) in TBST was used for blocking, and then the membrane was incubated overnight at $4{ }^{\circ} \mathrm{C}$ with primary antibody (1:1000). After washing and incubation, the membrane was incubated in TBST with secondary antibody (1:2000). Immunodetection was performed using the ECL Plus detection system (Sigma, USA) according to the manufacturer's instructions. The grey of the protein bands was measured using Image J software.

\section{MTT assay}

Cells were collected $48 \mathrm{~h}$ after transfection and seeded in 96-well plates at a concentration of $5 \times 10^{4}$ cells per well. MTT assay was applied for measuring cell proliferation after treatments with NC, miR-200c mimic, and miR-200c inhibitor. Briefly, $20 \mu \mathrm{L}$ of MTT reagent was added to the cells. After $3 \mathrm{~h}$ of incubation, the supernatant was removed, and $200 \mu \mathrm{L}$ of DMSO was added. The optical density of each well at $450 \mathrm{~nm}$ was detected after a 2-h incubation. Each assay was performed in triplicate.

\section{Dual luciferase reporter assay}

The binding site between miR-200c and AKT2 was predicted using Targetscan (http://www.targetscan. org/vert_71/). The 3'-untranslated region (UTR) of the AKT2 gene including the miR-200c binding target was amplified by PCR. The PCR amplification product of the AKT2 3'UTR was subcloned into the plasmid PGL3 vector (Promega, Madison, USA). The successfully identified vector was named AKT2-WT. The vector constructed from the mutant binding site sequence was named AKT2-MUT. To verify the binding site between the AKT2 and miR-200c, 5,637 cells in 96-well plates were cotransformed with miR-NC or miR-200c mimic and AKT2WTor AKT2-MUT using Lipofectamine 2000 (Invitrogen, United States). After $36 \mathrm{~h}$, the dual-luciferase reporter gene (firefly and Renilla) was measured. Firefly luciferase activity was normalized against Renilla luciferase activity.

\section{Tubule formation assay}

A tube formation assay was conducted as follows. Briefly, the 96-well plates were coated with $100 \mu \mathrm{L}$ of Matrigel (BD Biosciences, USA) per well and maintained at $37^{\circ} \mathrm{C}$ for $45 \mathrm{~min}$. Then, the cells were seeded into wells that were resuspended in $150 \mu \mathrm{L}$ of complete DMEM at a concentration of $5 \times 10^{5} / \mathrm{mL}$ and cultured for $24 \mathrm{~h}$ at $37{ }^{\circ} \mathrm{C}$. Pictures were captured under the microscope in bright field at $100 \times$ magnification. The number of tube branches and junctions were measured in three fields $(4 \times)$ using ChemiImager 5500 V2.03 software (Alpha Innotech, USA). The average was calculated by counting the branch number in three fields.

\section{Transwell assay}

Cell activity was measured through polycarbonate membrane Boyden chambers in a transwell apparatus (Costar, USA). Cells were first suspended in serum-free medium. Then, the suspended cells $\left(10^{5}\right)$ were added to the top chamber. The lower chamber was filled with $1 \mathrm{~mL}$ of DMEM containing $10 \%$ FBS. The cells on the top surface of the membrane were removed by a cotton swab after incubation $\left(24 \mathrm{~h}, 37^{\circ} \mathrm{C}\right)$. The migrated cells on the bottom surface of the membrane were fixed with methanol (100\%) and stained with crystal violet $(0.5 \%)$ for $2 \mathrm{~min}$. Then, the number of invasive cells was counted. The values for this experiment were obtained after counting three different fields in each membrane.

\section{Wound-healing assay}

After different treatments, cells were plated in a 6-well plate and cultured at $37{ }^{\circ} \mathrm{C}$. When the cells grew to $70 \%$ confluence, a wound was conducted along the centre of every well by a sterile $1-\mathrm{mL}$ pipette tip. Images were acquired after different incubation times $(0 \mathrm{~h}, 24 \mathrm{~h})$ at $37{ }^{\circ} \mathrm{C}(0 \mathrm{~h})$ with an inverted microscope (Olympus CKX 31 , Japan). Six fields for each group were chosen, and the cells 

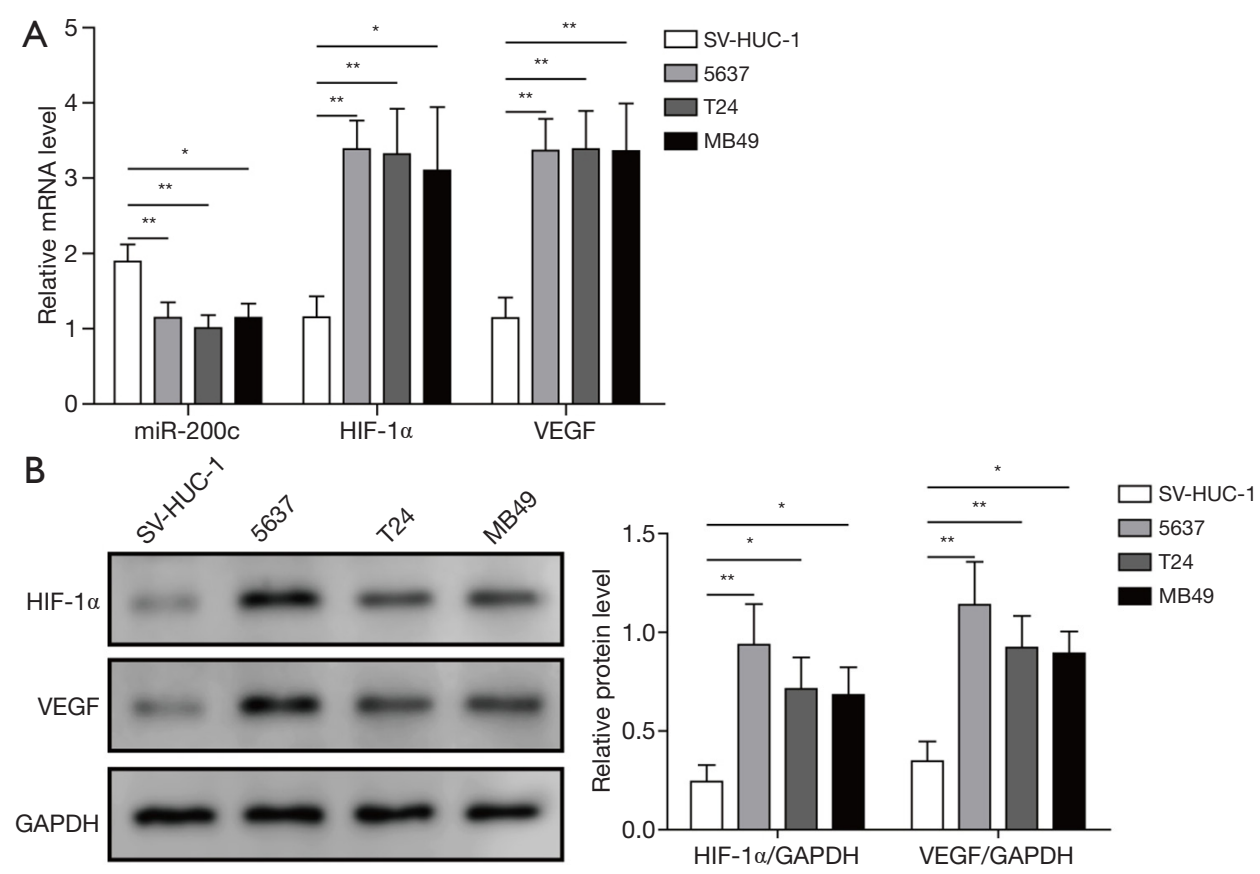

Figure 1 Expression of VEGF and HIF-1 $\alpha$ in bladder cancer cells. The expression of miR-200c, HIF-1 $\alpha$, and VEGF in SV-HUC-1, 5637, T24, and MB49, as measured by qPCR (A). The expression of HIF-1 $\alpha$, and VEGF in SV-HUC-1, 5637, T24, and MB49, as measured by western blotting (B). Values are displayed as the mean $\pm \mathrm{SD}$ from 3 independent experiments. ${ }^{*} \mathrm{P}<0.05$ or ${ }^{* *} \mathrm{P}<0.01$, compared with $\mathrm{SV}-\mathrm{HUC}-1$.

in the wound area were counted and analysed by ImageJ software.

\section{Statistical analysis}

Assays were performed in at least three independent experiments with one representative picture presented. Data are presented as the mean \pm standard deviation (SD). Statistical analysis was conducted using SPSS 24.0. A two-tailed Student's t-test was applied to assess the statistical significance of the difference between two independent groups. $\mathrm{P}$ values $<0.05$ were considered statistically significant.

\section{Results}

\section{Expression of HIF-1 $\alpha$ and VEGF in bladder cancer cells}

We measured the mRNA expression of miR-200c, VEGF, and HIF-1 $\alpha$ in SV-HUC-1, 5637, T24, and MB49 cells by qPCR, and the protein expression of VEGF and HIF$1 \alpha$ were also detected using western blotting. The results indicated that both the mRNA and protein expression of HIF-1 $\alpha$ and VEGF in bladder cancer cells (5637, T24, and MB49) was significantly higher than that in SV-HUC-1, a type of normal bladder epithelial cell line (Figure 1). However, the mRNA expression of miR-200c was markedly downregulated in bladder cancer cells compared with normal bladder epithelial cells (Figures $1 A$ ). These findings suggest that the expression of miR-200c in bladder cancer may be associated with abnormal expression of angiogenesis-related proteins.

\section{miR-200c affects bladder cancer cells by inbibiting angiogenesis}

Two bladder cancer cell lines, T24 and 5637, were chosen to study the influence of miR-200c on angiogenesis in bladder cancer cells. The mRNA expression of miR-200c in both cell lines was significantly higher after transfecting miR-200c mimic compared with the control group, and transfection with miR-200c inhibitor notably reduced the level of miR-200c (Figure 2A), indicating effective transfection in bladder cancer cell lines. Cell viability was measured after treatments with NC, miR-200c mimic, and miR-200c inhibitor. The results indicated that the cell viability of bladder cancer cells, 5637 and T24, increased markedly after miR-200c inhibition, but transfection with miR-200c mimic markedly reduced the proliferation 

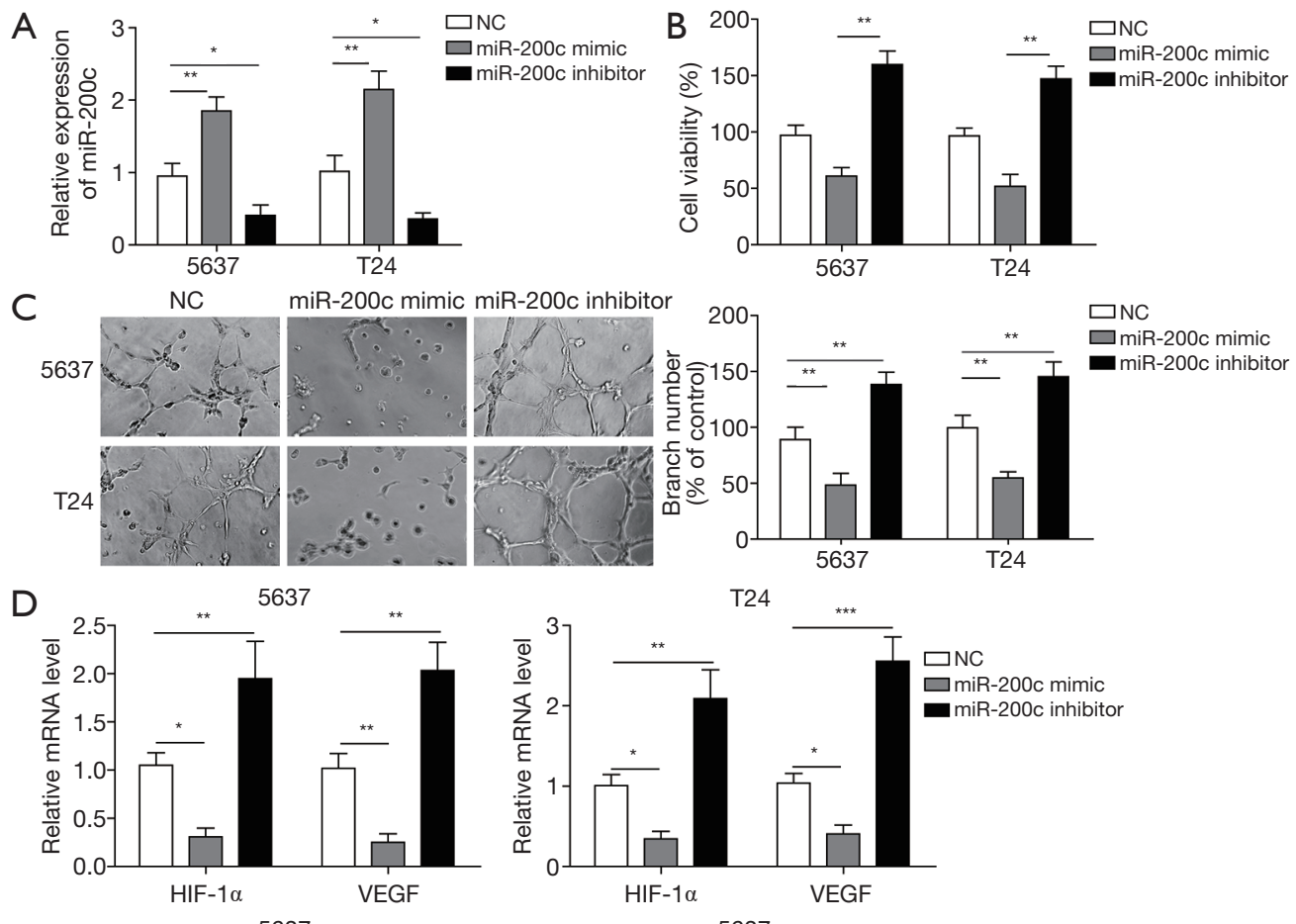

E
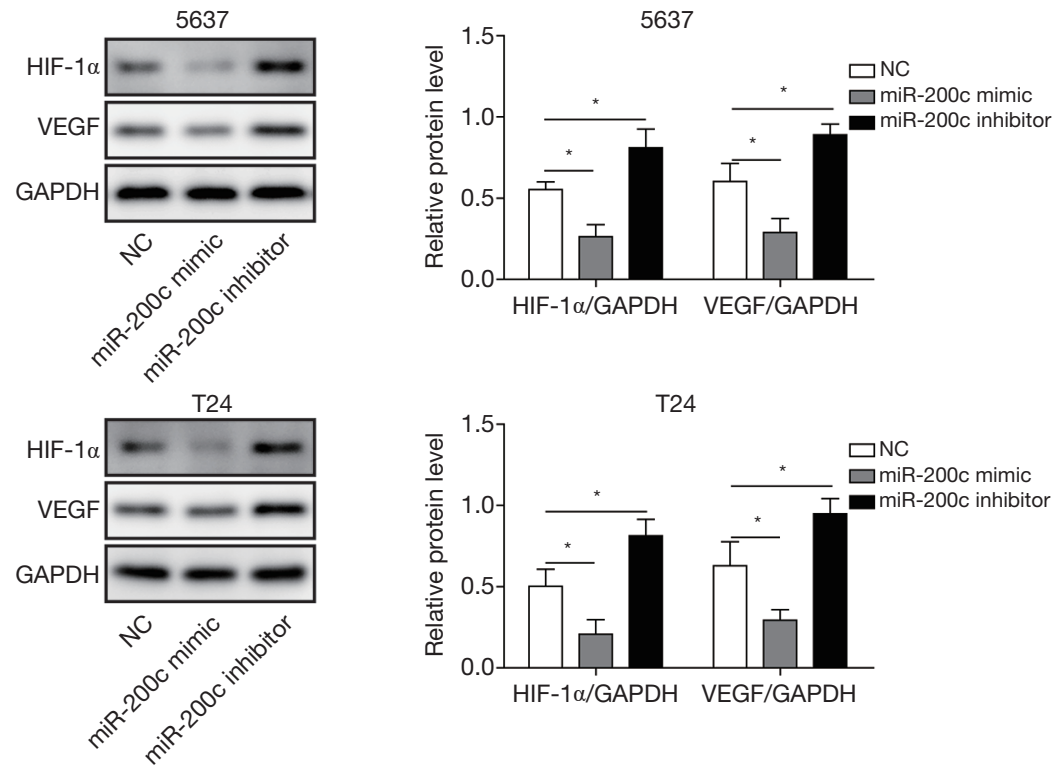

Figure 2 miR-200c affects bladder cancer cells by inhibiting angiogenesis. Transfection with miR-200c mimic or miR-200c inhibitor in 5637 and T24 cell lines (A); Measurement of cell viability after transfection with miR-200c mimic or miR-200c inhibitor (B); The angiogenesis was measured through tube formation assay after transfection with miR-200c inhibitor or miR-200c mimic (100x) (C); mRNA measurement of HIF-1 $\alpha$ and VEGF by qPCR after transfecting miR-200c mimic or miR-200c inhibitor (D); Protein measurement of HIF$1 \alpha$ and VEGF by western blotting after transfecting miR-200c mimic or miR-200c inhibitor (E). Values are displayed as the mean \pm SD from 3 independent experiments. ${ }^{*} \mathrm{P}<0.05$ or ${ }^{* *} \mathrm{P}<0.01$, ${ }^{* *} \mathrm{P}<0.001$ compared with the blank control and NC. 

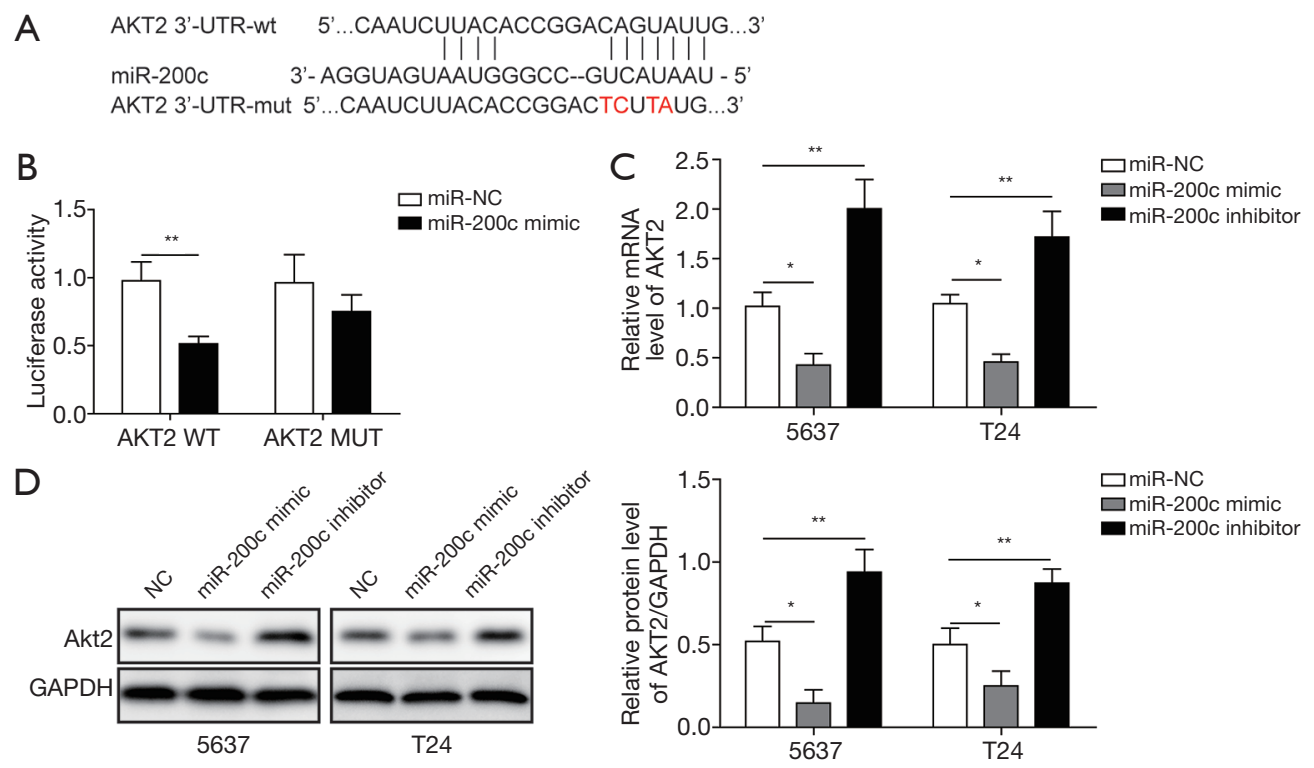

Figure 3 miR-200c affects the expression of Akt2. Binding sequence between miR-200c and the 3'-UTR of Akt2 transcripts (A); The dual luciferase activity assay showed binding affinity between miR-200c and Akt2 (B); mRNA measurement of Akt2 by qPCR after transfecting miR-200c mimic or miR-200c inhibitor (D); Protein measurement of Akt2 by western blotting after transfecting miR-200c mimic or miR200c inhibitor (E). Values are displayed as the mean $\pm \mathrm{SD}$ from 3 independent experiments. ${ }^{*} \mathrm{P}<0.05$ or ${ }^{* *} \mathrm{P}<0.01$, compared with the blank control and NC.

ability of bladder cancer cells (5637 and T24) (Figure 2B). We further performed a tubule formation test to detect the influence of different treatments on angiogenesis ability. After calculating the branch number, we found that transfecting the miR-200c mimic markedly inhibited angiogenesis (Figure 2C), but transfection with the miR$200 \mathrm{c}$ inhibitor produced the opposite effect. We also determined that transfection with miR-200c inhibitor significantly upregulated the protein and mRNA expression of HIF- $1 \alpha$ and VEGF, but miR-200c mimic markedly reduced their levels (Figure 2D,E). Therefore, miR-200c can negatively regulate angiogenesis in bladder cancer and further negatively regulate the expression of angiogenesisrelated proteins, such as HIF-1 $\alpha$ and VEGF.

\section{miR-200c affects the expression of Akt2}

Akt2 was considered a downstream target of miR-200c, and a luciferase assay was used to unfold the binding site between them. We predicted the binding sites between miR-200c and WT-Akt2 by bioinformatics. The wildtype 3'-UTR of Akt2 (WT-Akt2) contained the predicted miR-200c target sites, and the mutant-type 3'-UTR of Akt2 (MUT-Akt2) lacked the miR-200c binding sites.
WT-Akt2 and MUT-Akt2 were constructed (Figure 3A). Subsequently, we validated the binding site between miR200c and Akt2 using a dual luciferase reporter assay. The findings suggested that miR-200c mimics significantly suppressed the luciferase activity of the reporter gene, which contained the 3'-UTR of Akt2, compared with the miR-NC group. The suppression was stopped when miR200c binding sites were absent in the MUT-Akt2 group (Figure 3B). The expression of Akt2 was measured by qPCR and western blotting after transfection with miR-200c mimic or miR-200c inhibitor. We found that overexpression of miR-200c induced by transfecting miR-200c mimic markedly suppressed the mRNA and protein expression of Akt2 compared with the controls (Figure 3C,D). However, transfection with miR-200c inhibitor markedly upregulated the level of Akt2 (Figure 3C,D). Therefore, we believe that Akt2 is a target of miR-200c in bladder cancer and that miR-200c can modulate the expression of Akt2.

\section{Akt2 influences the expression of HIF-1 / /VEGF through mTOR}

Additionally, we further investigated if Akt2 could influence HIF-1 $\alpha$ /VEGF through mTOR. First, we transfected 
A

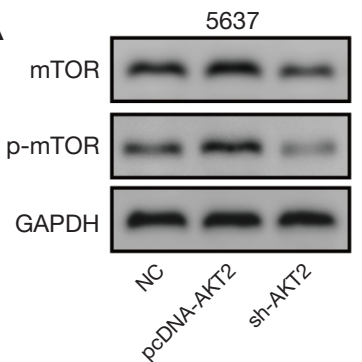

T24

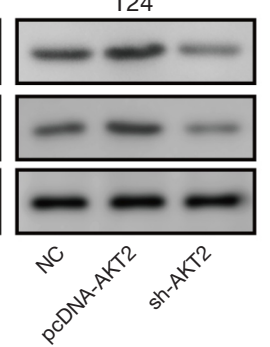

5637

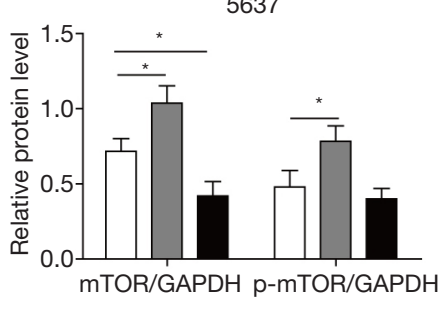

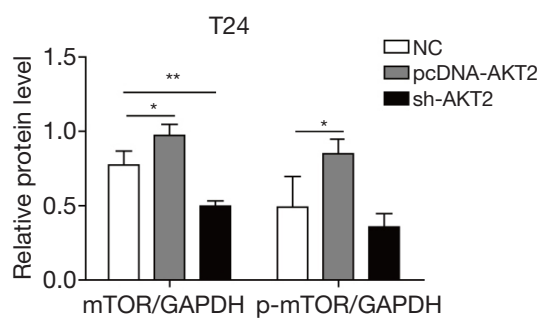

B
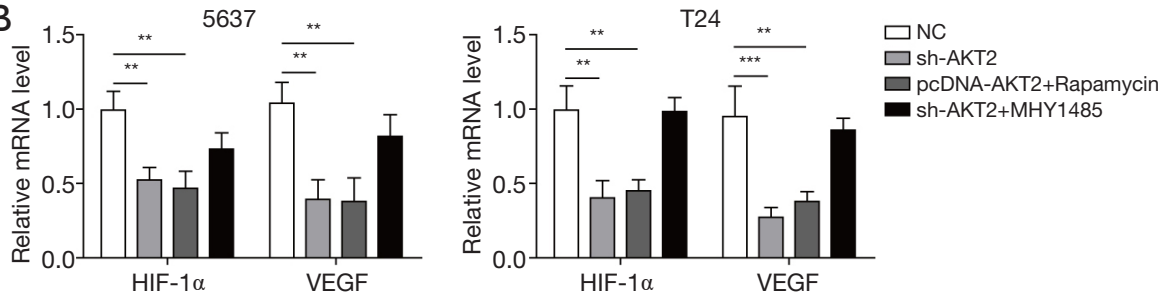

C
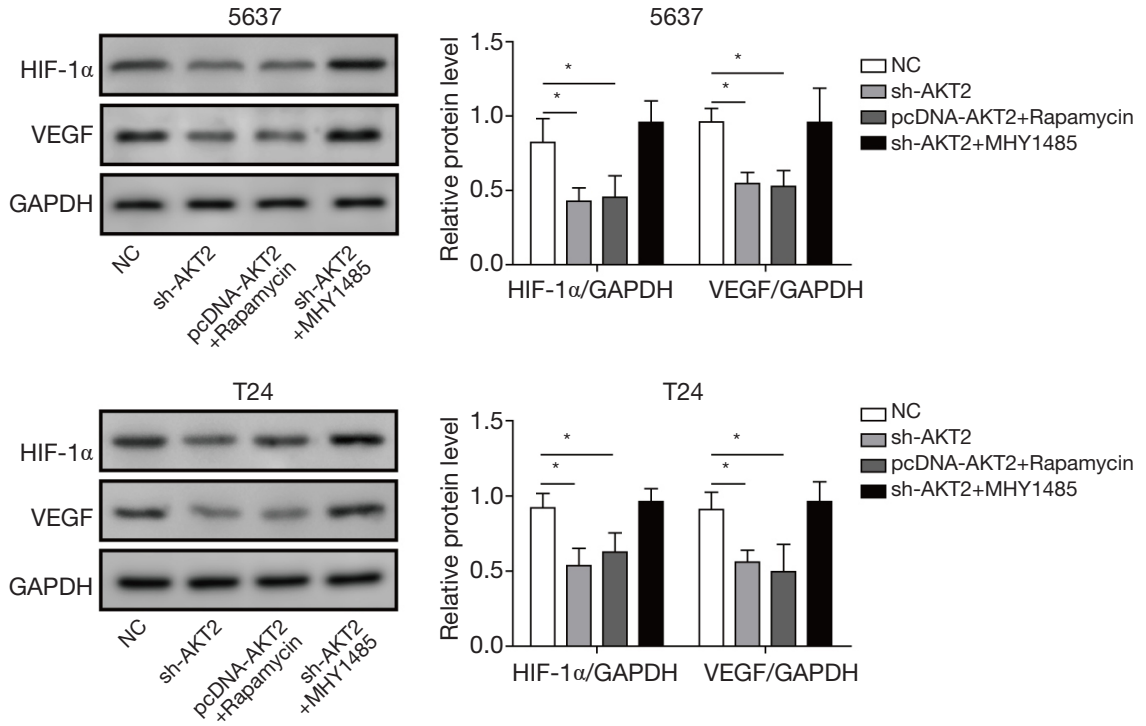

Figure 4 Akt2 influences the expression of HIF-1 $\alpha$ VEGF through mTOR. Protein measurement of mTOR and p-mTOR by western blotting after transfecting pcDNA-Akt2 and sh-Akt2 (A); mRNA measurement of VEGF and HIF-1 $\alpha$ by qPCR after treatment with shAkt2, pcDNA-Akt2 + Rapamycin, or sh-Akt2 + MHY1485 (B); Protein measurement of VEGF and HIF-1 $\alpha$ by western blotting after treatment with sh-Akt2, pcDNA-Akt2 + Rapamycin, or sh-Akt2 + MHY1485 (C). Values are displayed as the mean \pm SD from 3 independent experiments. ${ }^{*} \mathrm{P}<0.05$ or ${ }^{* *} \mathrm{P}<0.01,{ }^{* *} \mathrm{P}<0.001$ compared with the blank control and $\mathrm{NC}$.

pcDNA-Akt2 and sh-Akt2 in both the 5627 and T24 cell lines and proved that overexpression of Akt2 significantly increased the expression of mTOR and p-mTOR, but shAkt2 exerted the opposite effect (Figure 4A). Therefore, Akt 2 could influence the expression of mTOR. Then, we treated both cell lines with sh-Akt2, pcDNA-Akt2 + Rapamycin, and sh-Akt2 + MHY1485 and measured the protein and mRNA levels of HIF- $1 \alpha$ and VEGF. Rapamycin is the specific inhibitor of mTOR, and MHY1485 is the activator of mTOR. The expression of mTOR was markedly decreased after treatment with sh-Akt2 (Figure $4 A$ ). Compared with group NC, the protein and mRNA expression of HIF-1 $\alpha /$ VEGF were remarkably decreased in sh-Akt2 and pcDNA-Akt2 + Rapamycin. However, no significant difference was observed between group sh-Akt2 + MHY1485 and group NC (Figure 4B,C). These findings indicate that Akt2 might influence the expression of VEGF and HIF- $1 \alpha$ through mTOR. 
miR-200c affects angiogenesis by regulating Akt2/mTOR/ HIF-1a in bladder cancer cells

To determine whether miR-200c influences the expression of HIF- $1 \alpha$ and VEGF by regulating Akt $2 / \mathrm{mTOR}$, we treated two cell lines with miR-200c mimic, miR-200c mimic + pcDNA-Akt2, and miR-200c mimic + MHY1485. We proved that transfection with miR-200c mimic markedly decreased the expression of VEGF and HIF- $1 \alpha$ (Figure $5 A, B)$, but overexpression of Akt 2 or activation of mTOR could reverse the expression inhibition of HIF-1 $\alpha$ and VEGF induced by miR-200c (Figure $5 A, B$ ). These results indicate that miR-200c could influence the expression of VEGF and HIF- $1 \alpha$ through Akt $2 / \mathrm{mTOR}$.

Then, we investigated whether miR-200c influences the angiogenesis of HUVEC induced by bladder cancer through Akt $2 / \mathrm{mTOR}$. Compared with the inhibition effect of miR-200c mimic on branch numbers (Figure 5C), treatment with miR-200c mimic + pcDNA-Akt2 or miR200c mimic+MHY1485 markedly promoted angiogenesis (Figure 5C). Therefore, miR-200c could influence angiogenesis through Akt2/mTOR. Additionally, we detected the influence of miR-200c/Akt $2 / \mathrm{mTOR}$ on cell invasion and migration ability. The results indicated that transfection with miR-200c mimic markedly suppressed the invasion and migration of cells (Figure 5D,E), but miR-200c mimic + pcDNA-Akt2 or miR-200c mimic + MHY1485 significantly promoted migration and invasion of bladder cancer cells (Figure 5D,E), which was consistent with the results of both angiogenesis factor expression and angiogenesis tests. Therefore, miR-200c in bladder cancer affects the expression of HIF- $1 \alpha$ and VEGF by regulating Akt2 and further influences angiogenesis, and the migration and invasion of bladder cancer cells.

\section{Discussion}

Angiogenesis has been considered a key factor in the growth and progression of tumours $(22,23)$, and angiogenesis inhibition has been viewed as an important therapeutic strategy for the prevention of tumour expansion and metastasis (24). It was reported that miRNAs can regulate angiogenesis by affecting the expression of angiogenesisrelated genes $(25,26)$. However, the specific mechanism of angiogenesis in bladder cancer remains unknown.

Some miRNAs have been demonstrated to be involved in the development of bladder cancer. Downregulation of miR-430 enhanced the development of bladder cancer by upregulating C-X-C chemokine receptor type 7 (CXCR7) (27). miR-430 interacted with the $\mathrm{X}$-inactive specific transcript (XIST) to modulate the growth, migration, and invasion of bladder cancer (28). Meanwhile, miRNAs participate in angiogenesis in many types of tumours (29). It was reported that miR-200c could modulate pancreatic cancer endothelial cell proliferation, migration, and angiogenesis and that it was closely related to the tumourigenesis of other cancers (9). Therefore, we investigated the influence of miR-200c on the angiogenesis of bladder cancer. We proved that the expression of miR-200c was downregulated in bladder cancer cells, and miR-200c can negatively regulate the expression of the angiogenesis-related proteins VEGF and HIF- $1 \alpha$ and then affect the process of tubule formation. Therefore, miR-200c regulates angiogenesis in bladder cancer and is a potential therapeutic target.

Subsequently, we further demonstrated that miR-200c regulated angiogenesis in bladder cancer through Akt2. Activation of the Akt pathway is frequently reported in tumourigenesis. Overexpression of Akt2 has been observed in prostate and breast cancer cells $(30,31)$, and it could be regulated by several miRNAs, including miR-708, miR-203, and miR-200c (8). miR-200c plays a key role in osteosarcoma tumour growth and chemosensitivity by targeting Akt2 (32). In this study, we identified the binding site between miR-200c and Akt2 using a dual luciferase reporter assay, and we demonstrated that miR200c influenced angiogenesis by negatively regulating Akt2. PI3K/AKT/mTOR pathway has been believed to be closely linked with bladder cancer (33), and PI3K could be activated by cytokine receptors through a tyrosine-kinasedependent mechanism consisting of PI3KCA (PI3K- $\alpha$ ) and PI3KCB (PI3K- $\beta$ ) (34). Meanwhile, it was reported that NMIBC (Non-muscle invasive bladder cancer) patients with a PI3KCA mutation could have a better prognosis in the recurrence and progression of several cancers, including bladder cancer $(35,36)$. Therefore, whether patients with some alteration in PI3KCA have a better prognosis through Akt $2 / \mathrm{mTOR}$ is a promising research point. PI3KCA could activate the PI3K pathway through AKT1 phosphorylation and then block the methylation that EZH2 exerts on its targets $(37,38)$. EZH2 is a major molecule in the progression of bladder cancer and can regulate the levels of members of the miR-200 family, including miR-200c and miR-141 (39). Meanwhile, the EZH2/miR-200 axis was considered to regulate anti-tumour effects through STAT3 signalling, which has been believed to be closely linked with muscle invasive tumours (39). This evidence indicates that there 


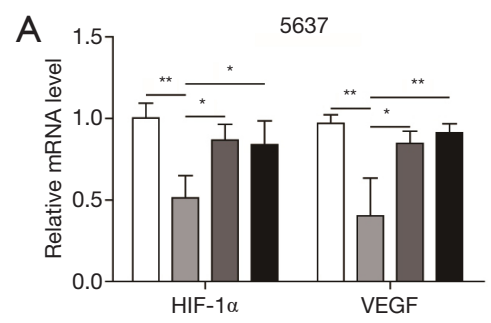

B
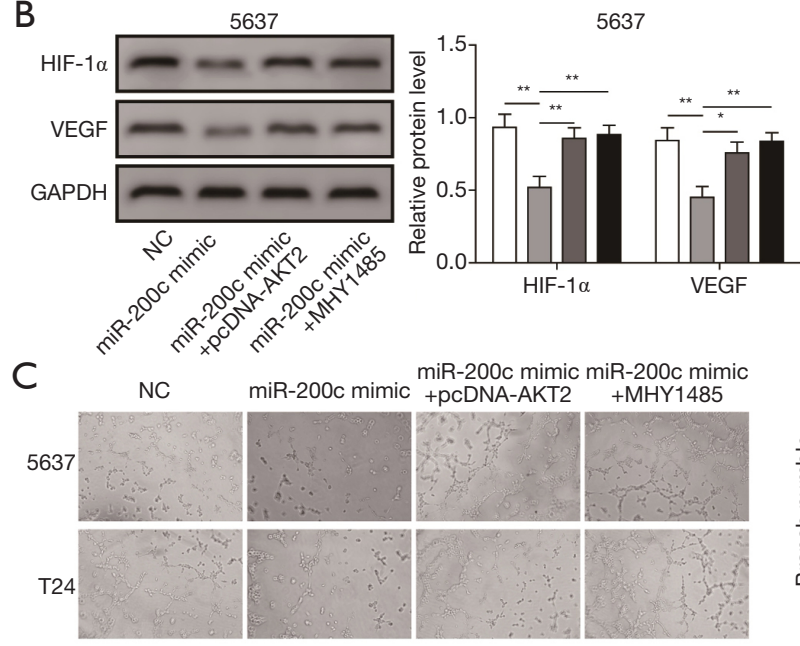

$\mathrm{HIF}-1 \alpha$

miR-200c mimic miR-200c mimic

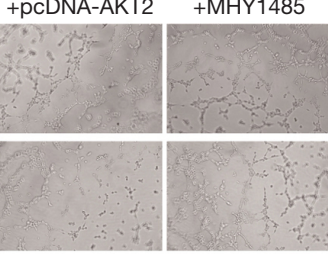

D NC miR-200c mimic miR-200c mimic

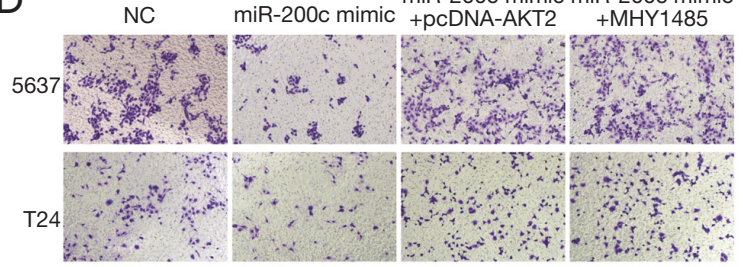

$\mathrm{E}$

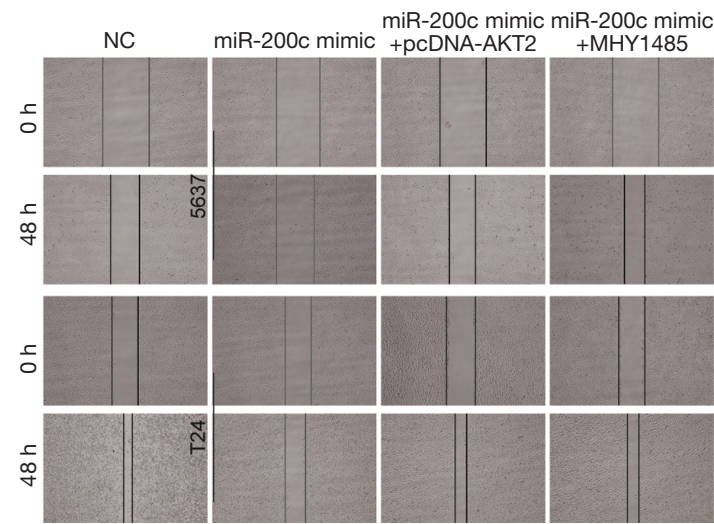

T24
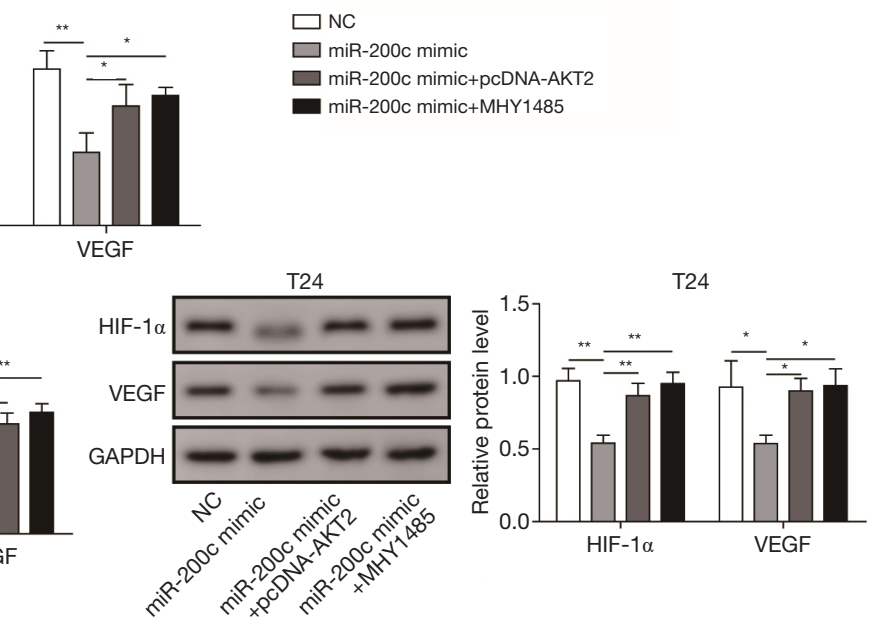
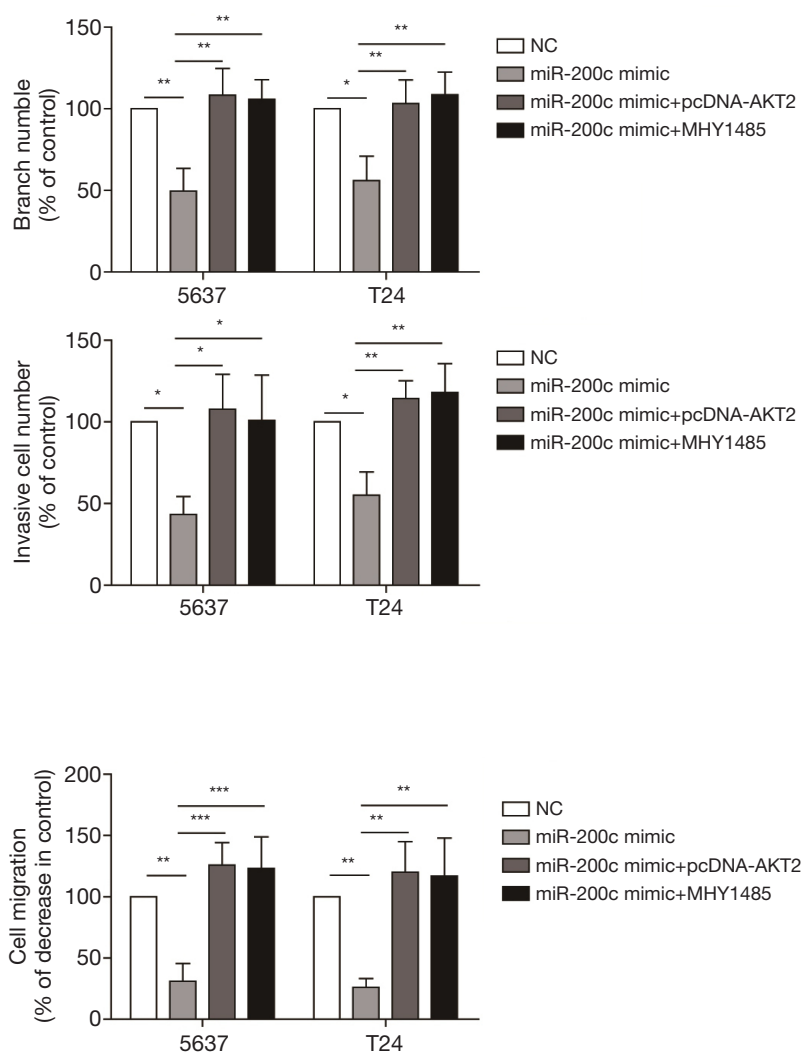

Figure 5 miR-200c affects angiogenesis by regulating Akt2/mTOR/HIF- $1 \alpha$ in bladder cancer cells. mRNA measurement of HIF- $1 \alpha$ and VEGF by qPCR after treatment with miR-200c mimic, miR-200c mimic + pcDNA-Akt2, or miR-200c mimic + MHY1485 (A); Protein measurement of HIF-1 $\alpha$ and VEGF by western blotting after treatment with miR-200c mimic, miR-200c mimic + pcDNA-Akt2, or miR-200c mimic + MHY1485 (B); The angiogenesis was measured using tube formation test after treatment with miR-200c mimic, miR-200c mimic + pcDNA-Akt2, or miR200c mimic + MHY1485 (100×) (C); Measurement of cell invasion by transwell assay after treatment with miR-200c mimic, miR-200c mimic + pcDNA-Akt2, or miR-200c mimic + MHY1485, cells stained with crystal violet (100x) (D); Measurement of cell migration by wound healing assay after treatment with miR-200c mimic, miR-200c mimic + pcDNA-Akt2, or miR-200c mimic + MHY1485 (100x) (E). Values are displayed as the mean $\pm \mathrm{SD}$ from 3 independent experiments. ${ }^{*} \mathrm{P}<0.05$ or ${ }^{* *} \mathrm{P}<0.01,{ }^{* * *} \mathrm{P}<0.001$ compared with the blank control and NC. 
may be a relationship of mutual regulation between AKT2 and EZH2. Therefore, the Akt $2 / \mathrm{mTOR} / \mathrm{HIF}-1 \alpha$ pathway may also be involved in the development of bladder cancer through the STAT3 pathway. Therefore, investigating the influence of Akt2/mTOR/HIF-1 $\alpha /$ STAT3 on the processes of drug resistance and intratumoural heterogeneity should be a promising research direction. Notch pathway has been viewed as a regulator of tumor angiogenesis, and activated STAT3 transfers from cytoplasm to nucleus and induces transcriptional activation of HIF-1 $\alpha$ (40). This study proved that HIF- $1 \alpha$ was up-regulated in bladder cancer cells, and the angiogenesis ability of bladder cancer cells was significantly higher in group HIF- $1 \alpha$ high expression, which is in line with previous study. Additionally, AKT2 is an important molecule of the insulin signalling pathway (41). Increased miR-200c expression in arteries from diabetic mice and patients with diabetes was observed, and miR-200c has been considered a new mediator of diabetic endothelial dysfunction (42). It has been found that miR-200c could mediate diabetic vascular dysfunction by up-regulating COX-2 expression (42). Therefore, this study may provide a novel treatment for bladder cancer patients combined with diabetes (type II), and AKT2 may also influence the muscleinvasive bladder carcinoma patients with diabetes (type II). The limitation of this study is that the cell lines we used were all invasive. Investigating other types of cell lines, such as high-risk or grade non-muscle-invasive cell lines, may help to confirm the conclusion of this study.

We explored the mechanism by which miR-200c influenced angiogenesis by regulating Akt2. The role of mTOR in tumour angiogenesis has been reported and related to the activity of the mTORC1 complex $(43,44)$. It was reported that blocking mTOR with rapamycin inhibits tumour angiogenesis by suppressing VEGF (45) and HIF-1 $\alpha$ (46). Meanwhile, Akt2 could affect angiogenesis by regulating mTOR (47). In this study, we found that miR-200c could influence the expression of HIF- $1 \alpha$ and VEGF by regulating Akt2/mTOR and could affect tubule formation and cell migration. Therefore, miR-200c may regulate the angiogenesis of bladder cancer through this mechanism described above.

In the present study, we demonstrated in vitro that miR200c could affect bladder cancer angiogenesis by regulating Akt $2 / \mathrm{mTOR} / \mathrm{HIF}-1 \alpha$. This study provides new insight into the progression, deterioration, and migration of bladder cancer. Meanwhile, therapeutic strategies targeting the Akt $2 / \mathrm{mTOR} / \mathrm{HIF}-1 \alpha$ pathway may be a promising treatment for bladder cancer.

\section{Acknowledgments}

Funding: This work was supported by Hunan Provincial Natural Science Foundation of China (2019JJ40442).

\section{Footnote}

Conflicts of Interest: All authors have completed the ICMJE uniform disclosure form (available at http://dx.doi. org/10.21037/tcr.2019.10.23). The authors have no conflicts of interest to declare.

Ethical Statement: The authors are accountable for all aspects of the work in ensuring that questions related to the accuracy or integrity of any part of the work are appropriately investigated and resolved.

Open Access Statement: This is an Open Access article distributed in accordance with the Creative Commons Attribution-NonCommercial-NoDerivs 4.0 International License (CC BY-NC-ND 4.0), which permits the noncommercial replication and distribution of the article with the strict proviso that no changes or edits are made and the original work is properly cited (including links to both the formal publication through the relevant DOI and the license). See: https://creativecommons.org/licenses/by-ncnd/4.0/.

\section{References}

1. Quan J, Pan X, Zhao L, et al. LncRNA as a diagnostic and prognostic biomarker in bladder cancer: a systematic review and meta-analysis. Onco Targets Ther 2018;11:6415-24.

2. Babjuk M, Bohle A, Burger M, et al. EAU Guidelines on Non-Muscle-invasive Urothelial Carcinoma of the Bladder: Update 2016. Eur Urol 2017;71:447-61.

3. Hou T, Zhou L, Wang L, et al. Leupaxin Promotes Bladder Cancer Proliferation, Metastasis, and Angiogenesis Through the PI3K/AKT Pathway. Cell Physiol Biochem 2018;47:2250-60.

4. Shi Q, Diao Y, Jin F, et al. Antimetastatic effects of Aidi on human esophageal squamous cell carcinoma by inhibiting epithelialmesenchymal transition and angiogenesis. Mol Med Rep 2018;18:131-8.

5. Xu R, Zhu X, Chen F, et al. LncRNA XIST/miR-200c regulates the stemness properties and tumourigenicity of human bladder cancer stem cell-like cells. Cancer Cell Int 
2018;18:41.

6. Pishkari S, Paryan M, Hashemi M, et al. The role of microRNAs in different types of thyroid carcinoma: a comprehensive analysis to find new miRNA supplementary therapies. J Endocrinol Invest 2018;41:269-83.

7. Bartel DP. MicroRNAs: target recognition and regulatory functions. Cell 2009;136:215-33.

8. Snowdon J, Zhang X, Childs T, et al. The microRNA-200 family is upregulated in endometrial carcinoma. PLoS One 2011;6:e22828.

9. Li L, Li B, Chen D, et al. miR-139 and miR-200c regulate pancreatic cancer endothelial cell migration and angiogenesis. Oncol Rep 2015;34:51-8.

10. Liu L, Qiu M, Tan G, et al. miR-200c inhibits invasion, migration and proliferation of bladder cancer cells through down-regulation of BMI-1 and E2F3. J Transl Med 2014;12:305.

11. Liu Y, Zhu ST, Wang X, et al. MiR-200c regulates tumor growth and chemosensitivity to cisplatin in osteosarcoma by targeting AKT2. Sci Rep 2017;7:13598.

12. Seiwert N, Neitzel C, Stroh S, et al. AKT2 suppresses prosurvival autophagy triggered by DNA double-strand breaks in colorectal cancer cells. Cell Death Dis 2017;8:e3019.

13. Foley NH, Bray IM, Tivnan A, et al. MicroRNA-184 inhibits neuroblastoma cell survival through targeting the serine/threonine kinase AKT2. Mol Cancer 2010;9:83.

14. Zhang J, Li G, Chen Y, et al. Metformin Inhibits Tumorigenesis and Tumor Growth of Breast Cancer Cells by Upregulating miR-200c but Downregulating AKT2 Expression. J Cancer 2017;8:1849-64.

15. Wang C, Che L, Hu J, et al. Activated mutant forms of PIK3CA cooperate with RasV12 or c-Met to induce liver tumour formation in mice via AKT2/mTORC1 cascade. Liver Int 2016;36:1176-86.

16. Liu LL, Lu SX, Li M, et al. FoxD3-regulated microRNA-137 suppresses tumour growth and metastasis in human hepatocellular carcinoma by targeting AKT2. Oncotarget 2014;5:5113-24.

17. Ptaszynska MM, Pendrak ML, Stracke ML, et al. Autotaxin signaling via lysophosphatidic acid receptors contributes to vascular endothelial growth factor-induced endothelial cell migration. Mol Cancer Res 2010;8:309-21.

18. Cheng Z, Fu J, Liu G, et al. Angiogenesis in JAK2 V617F positive myeloproliferative neoplasms and ruxolitinib decrease VEGF, HIF-1 enesis in JAK2 V617F positive cells. Leuk Lymphoma 2018;59:196-203.

19. Zhang J, Lu A, Li L, et al. p16 Modulates VEGF expression via its interaction with HIF-1alpha in breast cancer cells. Cancer Invest 2010;28:588-97.

20. Kitajima Y, Miyazaki K. The Critical Impact of HIF-1a on Gastric Cancer Biology. Cancers (Basel) 2013;5:15-26.

21. Mi C, Ma J, Shi H, et al. 4',6-dihydroxy-4methoxyisoaurone inhibits the HIF-1alpha pathway through inhibition of Akt/mTOR/p70S6K/4E-BP1 phosphorylation. J Pharmacol Sci 2014;125:193-201.

22. Zhao LN, Wang P, Liu YH, et al. MiR-383 inhibits proliferation, migration and angiogenesis of gliomaexposed endothelial cells in vitro via VEGF-mediated FAK and Src signaling pathways. Cell Signal 2017;30:142-53.

23. Furukawa K, Uwagawa T, Haruki K, et al. Nuclear factor kappaB activity correlates with the progression and prognosis of pancreatic cancer in a mouse model. Surg Today 2013;43:171-7.

24. Wang Z, Dabrosin C, Yin X, et al. Broad targeting of angiogenesis for cancer prevention and therapy. Semin Cancer Biol 2015;35 Suppl:S224-43.

25. Chen Y, Gao DY, Huang L. In vivo delivery of miRNAs for cancer therapy: challenges and strategies. Adv Drug Deliv Rev 2015;81:128-41.

26. Chang HC, Hsieh TH, Lee YW, et al. c-Myc and viral cofactor Kaposin B co-operate to elicit angiogenesis through modulating miRNome traits of endothelial cells. BMC Syst Biol 2016;10 Suppl 1:1.

27. Liu L, Zhao X, Zhu X, et al. Decreased expression of miR430 promotes the development of bladder cancer via the upregulation of CXCR7. Mol Med Rep 2013;8:140-6.

28. Xiong Y, Wang L, Li Y, et al. The Long Non-Coding RNA XIST Interacted with MiR-124 to Modulate Bladder Cancer Growth, Invasion and Migration by Targeting Androgen Receptor (AR). Cell Physiol Biochem 2017;43:405-18.

29. Landskroner-Eiger S, Moneke I, Sessa WC. miRNAs as modulators of angiogenesis. Cold Spring Harb Perspect Med 2013;3:a006643.

30. Arboleda MJ, Lyons JF, Kabbinavar FF, et al. Overexpression of AKT2/protein kinase Bbeta leads to up-regulation of beta1 integrins, increased invasion, and metastasis of human breast and ovarian cancer cells. Cancer Res 2003;63:196-206.

31. Saini S, Majid S, Shahryari V, et al. miRNA-708 control of CD44(+) prostate cancer-initiating cells. Cancer Res 2012;72:3618-30.

32. Attoub S, Arafat K, Hammadi NK, et al. Akt2 knockdown reveals its contribution to human lung cancer cell proliferation, growth, motility, invasion and endothelial cell tube formation. Sci Rep 2015;5:12759. 
33. Lv XY, Ma L, Chen JF, et al. Knockdown of DUXAP10 inhibits proliferation and promotes apoptosis in bladder cancer cells via $\mathrm{PI} 3 \mathrm{~K} / \mathrm{Akt} / \mathrm{m}$ TOR signaling pathway. Int J Oncol 2018;52:288-94.

34. Azab F, Vali S, Abraham J, et al. PI3KCA plays a major role in multiple myeloma and its inhibition with BYL719 decreases proliferation, synergizes with other therapies and overcomes stroma-induced resistance. Br J Haematol 2014;165:89-101.

35. Cousin S, Khalifa E, Crombe A, et al. Targeting ERBB2 mutations in solid tumors: biological and clinical implications. J Hematol Oncol 2018;11:86.

36. Tian S, Simon I, Moreno V, et al. A combined oncogenic pathway signature of BRAF, KRAS and PI3KCA mutation improves colorectal cancer classification and cetuximab treatment prediction. Gut 2013;62:540-9.

37. Cortelazzi B, Verderio P, Ciniselli CM, et al. Receptor tyrosine kinase profiles and human papillomavirus status in oropharyngeal squamous cell carcinoma. J Oral Pathol Med 2015;44:734-45.

38. Je EM, An CH, Yoo NJ, et al. Mutational analysis of PIK3CA, JAK2, BRAF, FOXL2, IDH1, AKT1 and EZH2 oncogenes in sarcomas. APMIS 2012;120:635-9.

39. Wang Y, Guo W, Li Z, et al. Role of the EZH2/miR200 axis in STAT3-mediated OSCC invasion. Int J Oncol 2018;52:1149-64.

40. Bridges E, Oon CE, Harris A. Notch regulation of tumor angiogenesis. Future Oncol 2011;7:569-88.

41. Kwon M, Eom J, Kim D, et al. Skeletal Muscle

Cite this article as: Wu SQ, He HQ, Kang Y, Xu R, Zhang L, Zhao XK, Zhu X. MicroRNA-200c affects bladder cancer angiogenesis by regulating the Akt2/mTOR/HIF-1 axis. Transl Cancer Res 2019;8(8):2713-2724. doi: 10.21037/tcr.2019.10.23
Tissue Trib3 Links Obesity with Insulin Resistance by Autophagic Degradation of AKT2. Cell Physiol Biochem 2018;48:1543-55.

42. Zhang H, Liu J, Qu D, et al. Inhibition of miR-200c Restores Endothelial Function in Diabetic Mice Through Suppression of COX-2. Diabetes 2016;65:1196-207.

43. Falcon BL, Barr S, Gokhale PC, et al. Reduced VEGF production, angiogenesis, and vascular regrowth contribute to the antitumor properties of dual mTORC1/mTORC2 inhibitors. Cancer Res 2011;71:1573-83.

44. Dodd KM, Yang J, Shen MH, et al. mTORC1 drives HIF1alpha and VEGF-A signalling via multiple mechanisms involving 4E-BP1, S6K1 and STAT3. Oncogene 2015;34:2239-50.

45. Sun S, Chen S, Liu F, et al. Constitutive Activation of mTORC1 in Endothelial Cells Leads to the Development and Progression of Lymphangiosarcoma through VEGF Autocrine Signaling. Cancer Cell 2015;28:758-72.

46. Wei J, Jiang H, Gao H, et al. Blocking Mammalian Target of Rapamycin (mTOR) Attenuates HIF-1alpha Pathways Engaged-Vascular Endothelial Growth Factor (VEGF) in Diabetic Retinopathy. Cell Physiol Biochem 2016;40:1570-7.

47. Martin KA, Merenick BL, Ding M, et al. Rapamycin promotes vascular smooth muscle cell differentiation through insulin receptor substrate-1/phosphatidylinositol 3-kinase/Akt2 feedback signaling. J Biol Chem 2007;282:36112-20. 